\title{
The Bilateral Sacral Origin of a Spinal Dural Arteriovenous Fistula: A
}

\section{Case Report}

\author{
Ehsan Mohammad Hosseini ${ }^{1}$, Alireza Rasekhi ${ }^{2}$, Keyvan Eghbal (iD) ${ }^{1}$, Abdolkarim Rahmanian ${ }^{1}$, Arash \\ Saffarrian ${ }^{1}$, Abbas Rakhsha ${ }^{1}$, Sulmaz Ghahramani (i) $^{3}$ and Mohammad Jamali ${ }^{1,}{ }^{*}$ \\ Neurosurgery Department, Shiraz University of Medical Sciences, Shiraz, Iran \\ ${ }^{2}$ Radiology Department, Shiraz University of Medical Sciences, Shiraz, Iran \\ ${ }^{3}$ Health Policy Research Center, Institute of Health, Shiraz University of Medical Sciences, Shiraz, Iran \\ "Corresponding author: Neurosurgery Department, Shiraz University of Medical Sciences, Nemazi Hospital, Shiraz, Iran. Email: mohammad.jamali.ns@gmail.com
}

Received 2021 April 13; Revised 2021 September 29; Accepted 2021 October 13.

\begin{abstract}
Spinal dural arteriovenous fistulas (SDAVFs) are characterized by an abnormal connection between a spinal radicular artery and a perimedullary vein, mainly fed by a radicular artery at the nerve root sleeve. Here, we describe the case of a 40-year-old woman, presenting with progressive weakness of the lower extremities and the sphincter. Thoracic magnetic resonance imaging (MRI) showed spinal cord edema and signal voids on the dorsal surface of the cord. Spinal angiography demonstrated a SDAVF with a nidus at the sacral level; the feeder of the arteriovenous fistula was a lateral sacral artery, as a branch of the internal iliac artery. The lateral sacral artery was subselectively catheterized, and SDAVF was embolized with 25\% n-butyl cyanoacrylate (NBCA) glue (glue: lipiodol ratio, 1:3). After embolization, no definite residual connection was visualized between the arterial and venous systems.
\end{abstract}

Keywords: Spine, Arteriovenous Fistula, Angiography

\section{Introduction}

Spinal arteriovenous fistulas (AVFs) and arteriovenous malformations (AVMs), as complex neurosurgical lesions, are significant causes of morbidity. They are responsible for 1-2\% of neurovascular pathologies (1). Patients typically have slowly progressive symptoms, such as pain, paresthesia, and myelopathy, and are often misdiagnosed with degenerative cervical or lumbar stenosis. These lesions can be classified in terms of pathophysiology, neuroimaging results, and neuroanatomy (2).

Spinal dural arteriovenous fistulas (SDAVFs) are the most common type of AVF lesions, accounting for up to $80 \%$ of all lesions identified (3). SDAVFs are characterized by an abnormal connection between a spinal radicular artery and a perimedullary vein, typically at the dural sleeve of the dorsal nerve root. However, other sites, such as the lateral sacral artery, are infrequently reported as the origin of $\operatorname{SDAVF}(4,5)$. It is evident that an uncommon origin of SDAVF may lead to a delayed diagnosis.

Arterialization of the medullary vein can lead to venous congestion of the spinal cord. As venous pressure increases, tissue perfusion decreases, along with vascular steal, resulting in ischemia and even hemorrhage in some cases (6). The primary diagnostic modalities for SDAVFs include magnetic resonance imaging (MRI) and spinal digital subtraction angiography (DSA). Besides, microsurgical obliteration, endovascular embolization, and radiosurgery are important therapeutic approaches (6).

\section{Case Presentation}

A 40-year-old woman was referred to our center with primary complaints of radicular lower back pain and slowly progressive paraparesis for nine months. Lumbosacral MRI and spinal DSA had been performed in another center for non-diagnostic purposes. In thoracic MRI, spinal cord edema from T6 to T12 (maximum edema at T11), as well as tortuous signal voids on the dorsal surface of the spinal cord, was detected.

After the patient was referred to our center, in her physical examination, bilateral paraparesis (bilateral muscle power was 3 out of 5 score) and increased deeptendon reflexes (3+ DTR) were observed. Notably, no urinary or fecal incontinence was observed. The patient underwent thoracic MRI, which showed spinal cord edema and signal voids on the dorsal surface of the cord (Figure 1). Following 
spinal angiography via injections into the subclavian, vertebral, and iliac arteries, computed tomography (CT) angiography of the thoracic aorta and spinal artery was performed, which was not diagnostic (Figure 1).

After three days, the patient's symptoms deteriorated, and complete paraplegia and urinary incontinence occurred. Emergency laminectomy and durotomy from T9 to L1 were also carried out. Although a tortuous, engorged vein was seen on the dorsal surface of the spinal cord, no fistula or direct connection was detected between the radicular artery and the perimedullary vein (Figure 2).

Moreover, no clinical improvement was seen after surgery. After two days, repeated spinal angiography indicated a dural AV fistula with a nidus at the sacral level, fed by the lateral sacral branch on both sides of the internal iliac artery with a slow venous drainage, indicative of cephalad extension into the T8 level. The lateral sacral artery was subselectively catheterized with a Marathon microcatheter (Medtronic, Minneapolis, MN, USA). After proper positioning in the left and right lateral sacral arteries responsible for supplying the lesion, DAVF was confirmed by DSA. Next, 25\% n-butyl cyanoacrylate (NBCA) glue (glue: lipiodol ratio, 1:3) was injected into the fistula and its venous segment under fluoroscopic visualization. After embolization with cyanoacrylate glue, no definite residual connection was visualized between the arterial and venous systems. There was no complication after embolization (Figure 3).

Thoracolumbar MRI was repeated one day after embolization, suggesting no signs of signal voids or tortuous veins (Figure 4). Two days after embolization, some clinical improvement was observed. Five days later, the muscle strength of the left and right lower limbs was 3/5 and 2/5, respectively.

\section{Discussion}

Spinal dural AVFs and other spinal vascular malformations are rare disorders, which are usually misdiagnosed; therefore, comprehensive and precise physical examination and clinical suspicion are essential for a timely diagnosis. Kim and Spetzler classified these lesions into six types: extradural AVFs, intradural dorsal AVFs, intradural ventral AVFs, extradural/intradural AVMs, intramedullary AVMs, and conus medullaris AVMs (2).

SDAVF, an intradural dorsal lesion according to Kim and Spetzler's classification, is responsible for $80 \%$ of all spinal vascular malformations and is usually located within the dura at the level of the intervertebral foramen. The fistula is commonly located in the thoracic and lumbosacral regions of the spinal column, while it is less frequently found in the cervical region. Moreover, its feeding artery originates from the radiculomeningeal, intercostal, and lumbar arteries, with venous drainage through the dilated radicular and/or perimedullary veins (7).

MRI is a sensitive modality for the diagnosis of SDAVFs, that shows increased T2 signals in the spinal cord and some of the perimedullary flow voids (8). Kiyosue et al. investigated 207 patients with spinal vascular malformations. Overall, 108 cases were diagnosed with SDAVFs, mostly fed by the radiculomeningeal artery; however, none of them were fed by the iliac arteries (9). In another study by Kang et al., the average time of diagnosis was 6.5 months, and the majority of patients showed myelopathy in their physical examinations at the time of diagnosis (10).

Microsurgical obliteration and endovascular embolization are the primary treatments for SDAVFs. Microsurgery contributes to definitive treatment, with a lower failure rate than embolization. In recent years, advances in embolic agents, imaging techniques, and microcatheter devices have resulted in growing interest in endovascular lesion embolization (10). Besides, the type and location of the fistula and the angioarchitecture of the lesion can affect the decision-making process for treatment.

Embolization of particles, such as polyvinyl alcohol (PVA), has been largely abandoned in the treatment of SDAVFs because of their high recanalization rate. NBCA is the main embolic agent used in the treatment of spinal vascular lesions (11). It is considered safe for the treatment of SDAVFs (12), with an acceptable embolization success rate compared to embolization with Onyx and PVA (8). Moreover, Alvarado et al. reported two cases of SDAVFs in their study. In the first case, the left sacral artery was found as the origin of fistula, embolized with NBCA. In the other patient, the right sacral artery was the feeder of fistula, for which endovascular embolization was carried out with ethylene vinyl alcohol (Onyx) (13).

There are reported cases of the unilateral sacral artery as the origin of SDAVFs $(4,7,14,15)$. However, SDAVFs, fed by the bilateral lateral sacral artery, have been rarely reported (16). In this study, we reported a dorsal type of SDAVF, fed bilaterally by the lateral sacral arteries of the internal iliac artery. These fistulas are mainly fed by a radicular artery at the nerve root sleeve. In our patient, the feeder originated from the lateral sacral artery as a branch of the internal iliac artery, for which complete embolization was performed with NBCA.

In conclusion, it is essential to perform selective spinal angiography of thoracic and lumbar intercostal arteries in patients with SDAVFs. If a fistula is not detected, injection into the subclavian, vertebral, external carotid, and iliac arteries is necessary. 

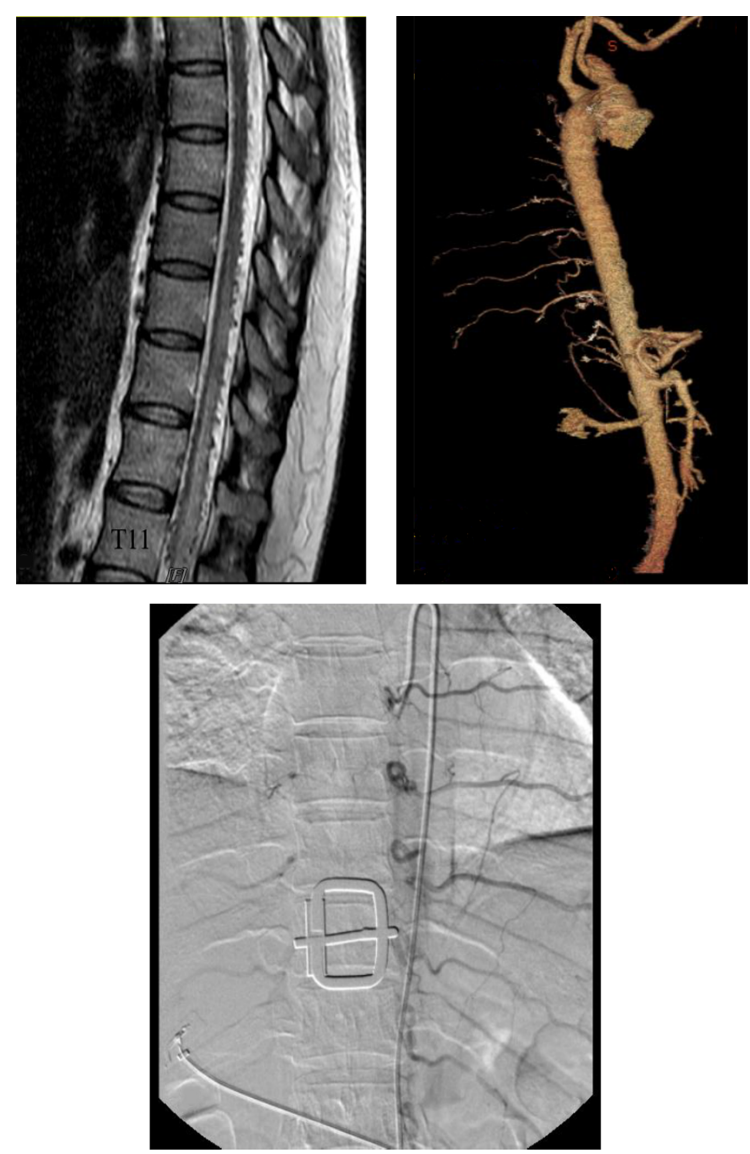

Figure 1. A, Sagittal T2-weighted MRI shows spinal cord edema, as well as dilated and tortuous signal voids; B, CT angiography of the thoracic aorta and spinal artery; C, Spinal

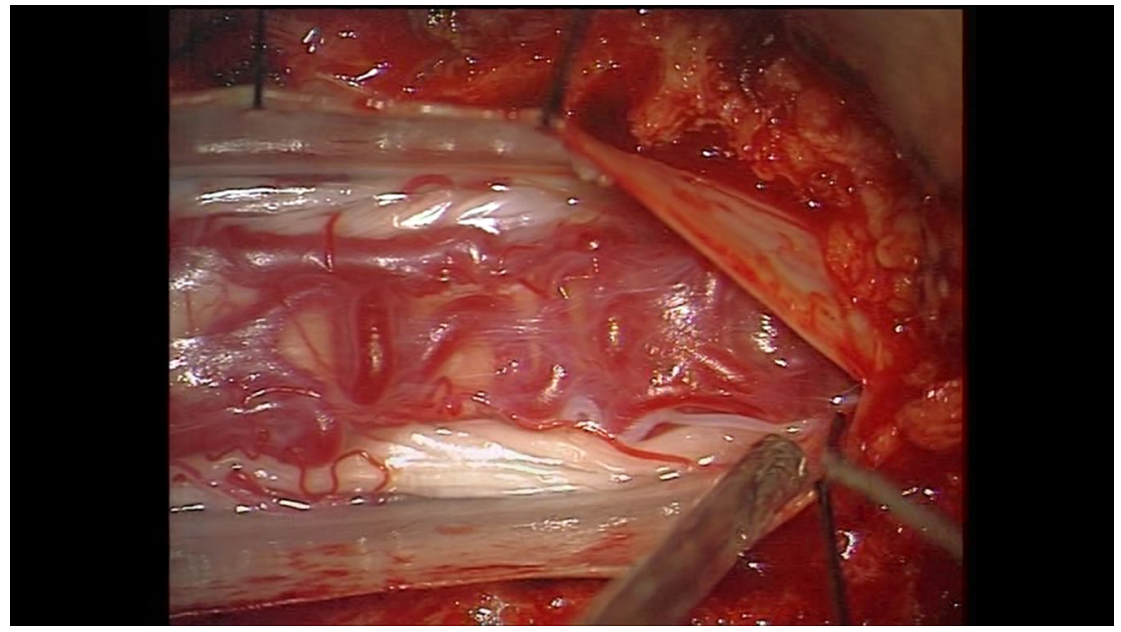

Figure 2. Intraoperative imaging after durotomy shows tortuous and engorged veins in the dorsal surface of the spinal cord 

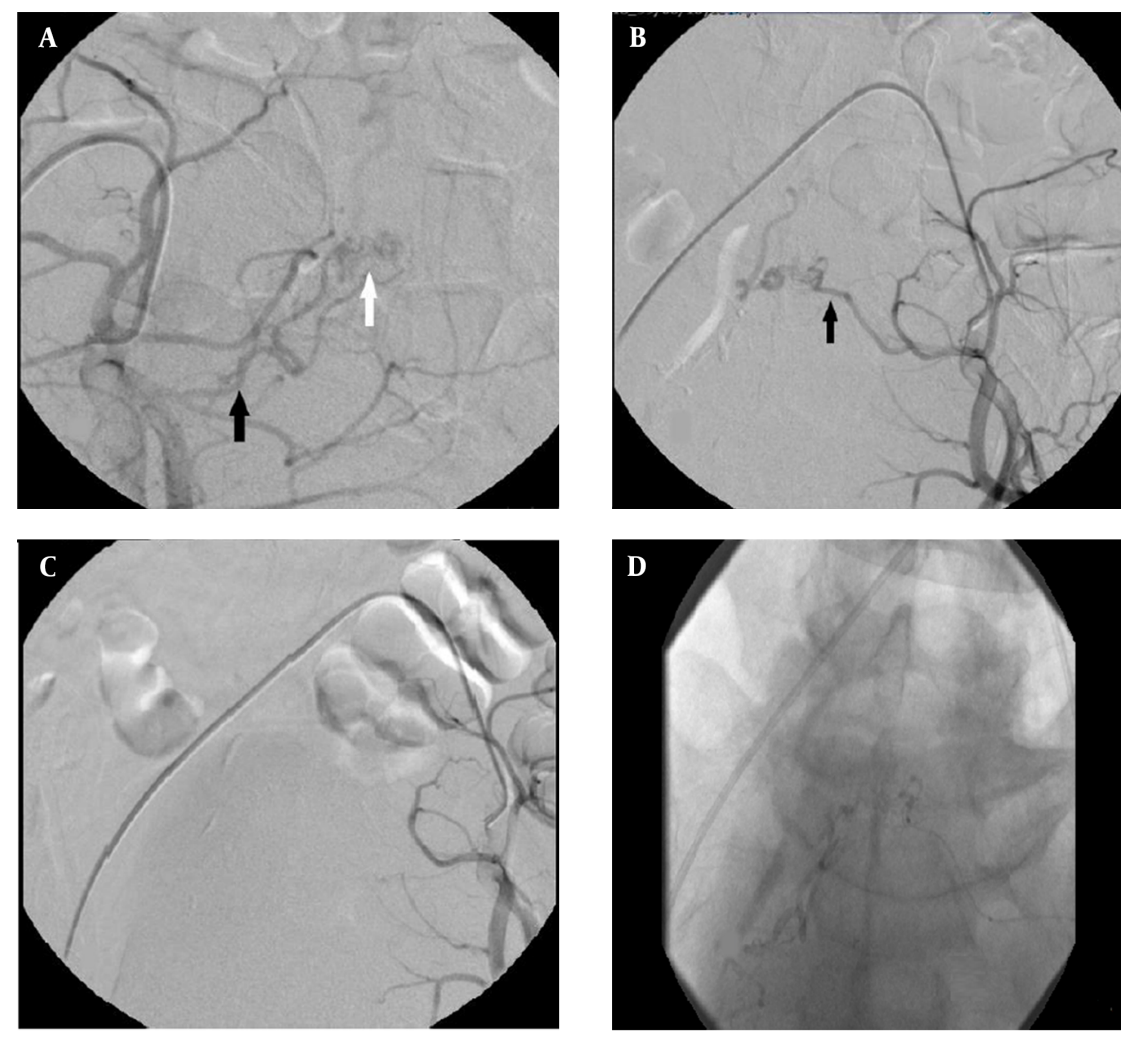

Figure 3. Spinal angiography; A, Injection into the right internal iliac artery (black arrow: feeder from the superior branch of the lateral sacral artery; white arrow: a nidus in the sacral area); B, Angiography of the left internal iliac artery shows a fistula from the lateral sacral artery; C, After embolization, no fistula was seen; D, The final image demonstrates n-BCA glue cast formation

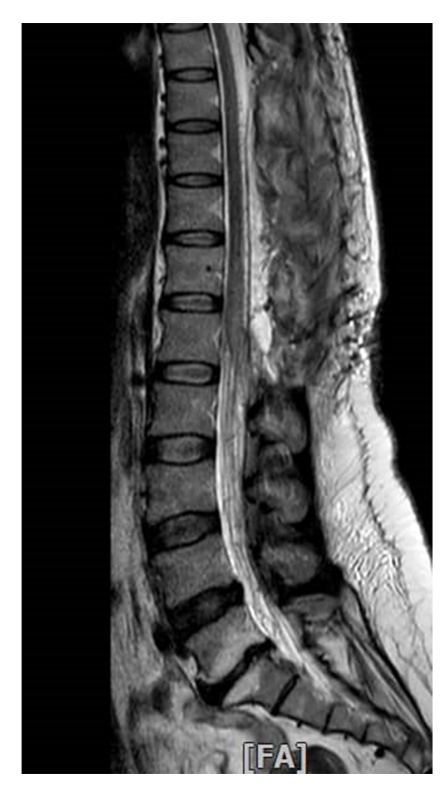

Figure 4. Sagittal T2-weighted MRI after laminectomy and embolization indicates diminished spinal cord edema and no signal voids 


\section{Acknowledgments}

We would like to thank the patient and her family for their cooperation.

\section{Footnotes}

Authors' Contribution: Study conception and design: M.J., A.RAS., K.E., A.R., A.S., and A.RAH.; Interpretation of data and drafting of the manuscript: E.MH., M.J., and S.GH.; and critical revision of the manuscript for important intellectual content: all authors.

Conflict of Interests: The authors declare no conflicts of interest.

Funding/Support: The authors received no funding for the publication of this study.

Informed Consent: Informed consent was obtained from the patient. All data were collected anonymously.

\section{References}

1. Fox S, Hnenny L, Ahmed U, Meguro K, Kelly ME. Spinal dural arteriovenous fistula: a case series and review of imaging findings. Spinal Cord Ser Cases. 2017;3(1):1-6. doi: 10.1038/scsandc.2017.24. [PubMed: 28690870]. [PubMed Central: PMC5498824].

2. Kim LJ, Spetzler RF. Classification and surgical management of spinal arteriovenous lesions: arteriovenous fistulae and arteriovenous malformations. Neurosurgery. 2006;59(5 Suppl 3). doi: 10.1227/01.NEU.0000237335.82234.CE. [PubMed: 17053603].

3. Takai K, Taniguchi M. Comparative analysis of spinal extradural arteriovenous fistulas with or without intradural venous drainage: a systematic literature review. Neurosurg Focus. 2012;32(5). E8. doi: 10.3171/2012.2.FOCUS1216. [PubMed: 22537134].

4. Gioppo A, Farago G, Giannitto C, Caputi L, Saladino A, Acerbi F, et al. Sacral dural arteriovenous fistulas: a diagnostic and therapeutic challenge - single-centre experience of 13 cases and review of the literature. J Neurointerv Surg. 2018;10(4):415-21. doi: 10.1136/neurintsurg2017-013307. [PubMed: 29025963].
5. Park KW, Park SI, Im SB, Kim BT. Spinal dural arteriovenous fistula with supply from the lateral sacral artery-case report and review of literature. J Korean Neurosurg Soc. 2009;45(2):115-7. doi: 10.3340/jkns.2009.45.2.115. [PubMed: 19274124]. [PubMed Central: PMC2651549].

6. Rangel-Castilla L, Russin JJ, Zaidi HA, Martinez-Del-Campo E, Park MS, Albuquerque FC, et al. Contemporary management of spinal AVFs and AVMs: lessons learned from 110 cases. Neurosurg Focus. 2014;37(3). E14. doi: 10.3171/2014.7.FOCUS14236. [PubMed: 25175433].

7. Nishio A, Ohata K, Takami T, Nishikawa M, Hara M. Atypical spinal dural arteriovenous fistula with supply from the lateral sacral artery. J Clin Neurosci. 2007;14(1):65-8. doi: 10.1016/j.jocn.2005.10.020. [PubMed: 17092723].

8. Sivakumar W, Zada G, Yashar P, Giannotta SL, Teitelbaum G, Larsen DW. Endovascular management of spinal dural arteriovenous fistulas. A review. Neurosurg Focus. 2009;26(5). E15. doi: 10.3171/2009.2.FOCUS098. [PubMed: 19408993].

9. Kiyosue H, Matsumaru Y, Niimi Y, Takai K, Ishiguro T, Hiramatsu M, et al. Angiographic and Clinical Characteristics of Thoracolumbar Spinal Epidural and Dural Arteriovenous Fistulas. Stroke. 2017;48(12):3215-22. doi: 10.1161/STROKEAHA.117.019131. [PubMed: 29114089]. [PubMed Central: PMC5704665].

10. Kang MS, Kim KH, Park JY, Kuh SU, Chin DK, Jin BH, et al. Comparison of Endovascular Embolization and Surgery in the Treatment of Spinal Intradural Dorsal Arteriovenous Fistulas. World Neurosurg. 2019;122:e1519-27. doi: 10.1016/j.wneu.2018.11.093. [PubMed: 30468927].

11. Flores BC, Klinger DR, White JA, Batjer HH. Spinal vascular malformations: treatment strategies and outcome. Neurosurg Rev. 2017;40(1):1528. doi: 10.1007/s10143-016-0713-z. [PubMed: 27075861].

12. Baranoski J, Catapano J, Cole T, Majmundar N, Hendricks B, Wilkinson D, et al. E-143 Embolization of spinal dural arteriovenous fistula: nBCA is superior to onyx embolization.J Neurointerv Surg. 2020;12 (Suppl1). doi: 10.1136/neurintsurg-2020-SNIS.175.

13. Alvarado AM, Haussen DC, Ebersole K, Nogueira RG, Abraham MG. Embolization of Sacral Dural Arteriovenous Fistulas: A Case Series and Literature Review. Interv Neurol. 2017;6(1-2):73-81. doi: 10.1159/000454929. [PubMed: 28611837]. [PubMed Central: PMC5465750].

14. Park KW, Rhim SC, Suh DC, Roh SW. Spinal dural arteriovenous fistula from lateral sacral artery. J Korean Neurosurg Soc. 2003;34(3):258-61.

15. Mull M, Schubert GA, Obersheimer J, Jablawi F. Unique Angioarchitecture of Sacral Dural Arteriovenous Fistula. World Neurosurg. 2020;133:25-8. doi:10.1016/j.wneu.2019.09.078. [PubMed: 31550539].

16. Lerner DJ, Samson M, Holloway W, Akhtar N. Spinal Dural Arteriovenous Fistula with Bilateral Lateral Sacral Arterial Supply and Embolization. Kans J Med. 2013;6(1):21-4. doi: 10.17161/kjm.v6i1.11431. 